\title{
P078: Epidemiology of extended-spectrum beta- lactamase-producing enterobacteriaceae (ESBL-E) during an epidemic, with screening of patients and healthcare workers
}

\author{
A Agostinho $^{\text {* }}$, G Jourdan², G Renzi ${ }^{3}$, C Bonfillon ${ }^{4}$, P Hoffmeyer ${ }^{2}$, S Harbarth ${ }^{1}$, I Uçkay ${ }^{1}$ \\ From 2nd International Conference on Prevention and Infection Control (ICPIC 2013) \\ Geneva, Switzerland. 25-28 June 2013
}

\section{Objectives}

To determine the nosocomial acquisition rate of ESBL-E among patients and healthcare workers (HCWs) during an epidemic (March 2009 to Nov 2010) in an orthopaedics ward at HUG.

\section{Methods}

Universal screening made by anal swab of all patients on admission and every 2 weeks if screening remained negative. 49 samples were collected from $41 \mathrm{HCW}$ and 60 environmental samples were analysed. Molecular typing was performed on all ESBL-E isolates. If there was more than $97.5 \%$ similarity, strains were considered identical.

\section{Results}

Between March 2009 and November 2010, 1'531 admissions occurred to the orthopaedic ward (12'401 patientdays; length of stay of 27 days). Among 565 anal swabs, ESBL-E were detected in 204 samples from 45 patients.

The ESBL-E found were E. coli $(\mathrm{n}=39)$, Klebsiella pneumoniae $(\mathrm{n}=10)$, Enterobacter spp $(\mathrm{n}=8)$, Citrobacter $\operatorname{spp}(\mathrm{n}=2)$, Morganella morganii $(\mathrm{n}=2)$, and Proteus vulgaris $(\mathrm{n}=1)$. Two different ESBL-E strains were detected in 6 patients, and 3 others carried three distinct isolates. The ESBL-E transmitted were E. coli (14 patients), K. pneumoniae (3 patients) and both in 2 patients.

Identical ESBL-E species with epidemiological links were found in 25 cases. Only 9 of these were attributable to the unit. Most positive patients (96\% [43/45]) were colonized asymptomatically with ESBL-E.

Infection Control Program, Geneva University Hospitals, Geneva, Switzerland Full list of author information is available at the end of the article
Among HCWs, 6 samples (12\%) were positive. Transmission was only observed between patients, not HCWs.

None of the environmental samples revealed presence of ESBL-E.

\section{Conclusion}

Transmission of ESBL-E strains was only observed between patients. No transmission between HCWs and patients occurred. HCW screening and environmental sampling is not useful during ESBL-E carriage outbreaks.

The main ESBL-E transmitted was E. coli.

ESBL-E transmission can occur in units with extended length of stay, questioning the new Swiss policy of abandoning contact precautions for E. coli-ESBL carriers.

\section{Disclosure of interest}

None declared.

\section{Author details}

'Infection Control Program, Geneva University Hospitals, Geneva, Switzerland. ${ }^{2}$ Orthopaedic Surgery, Geneva University Hospitals, Geneva, Switzerland.

${ }^{3}$ Central Bacteriology Laboratory, Geneva University Hospitals, Geneva, Switzerland. ${ }^{4}$ Occupational Medicine Service, Geneva University Hospitals, Geneva, Switzerland.

Published: 20 June 2013

\section{doi:10.1186/2047-2994-2-S1-P78}

Cite this article as: Agostinho et al:: P078: Epidemiology of extended-spectrum beta-lactamase-producing enterobacteriaceae

(ESBL-E) during an epidemic, with screening of patients and healthcare workers. Antimicrobial Resistance and Infection Control 2013 2(Suppl 1):P78. 